\title{
American Board of Family Medicine Introduces Important Enhancements to Maintenance of Certification for Family Physicians
}

The American Board of Family Medicine (ABFM) has always affirmed that it would listen carefully to feedback and concerns from Diplomates, and would continuously make improvements to the Maintenance of Certification for Family Physicians (MC-FP), simplifying and streamlining the process, targeting it to practice needs, and creating flexibility in how the MC-FP requirements are met.

As of 2011, the ABFM introduced several important enhancements to the MC-FP. These changes will affect only those Diplomates who are certifying or recertifying in 2011 and beyond. The first of these enhancements is that, beginning with those Diplomates who certify or recertify in the summer of 2011, the examination will be temporally unlinked from the MC-FP cycle. At the present time, MC-FP culminates with the administration of the examination at the end of the cycle; with this change the examination may be taken at any point during the MC-FP cycle, and the results will be valid for up to 10 years.

Secondly, Diplomates certifying in 2011 and after will be allowed to choose how they wish to complete their part II (Self-Assessment modules [SAMs]) and part IV (Performance in Practice modules, Methods in Medicine modules, or approved alternatives) requirements during each 3 -year stage. Each module will be assigned a point value, and each Diplomate will need to accumulate 50 MC-FP points every 3 years. How this is accomplished is up to the Diplomate, as long as the combination of completed activity includes at least one ABFM SAM and one part IV quality improvement activity. The basic requirements will be the same for each 3-year period.
In addition, Diplomates must continuously meet the requirements of all 4 parts of MC-FP to remain certified. With this new format in place, each Diplomate will simply need to meet 4 requirements during each 3-year stage of the MC-FP cycle to maintain certification.

1. Maintain medical license and keep current record with ABFM (part I).

2. Complete part II and part IV activities totaling 50 MC-FP points, with a minimum one ABFM SAM and one part IV quality improvement activity (Diplomates will continue to receive continuing medical education [CME] credits for this activity).

3. Meet ABFM CME requirements of $150 \mathrm{CME}$ credits per stage (part II).

4. Perform successfully on the examination every 10 years (part III).

Lastly, the ABFM is awaiting approval from the American Board of Medical Specialties to begin issuing certificates without end dates beginning in the summer. The validity of the new certificates without end dates will be continuous as long as the requirements for the MC-FP program are adhered to and the Diplomate successfully completes the examination every 10 years.

Again, these enhancements will only affect those Diplomates certifying or recertifying in 2011 and beyond. For questions regarding these changes or the MC-FP, Diplomates may contact the ABFM Support Center at $877-223-7437$ or via E-mail at help@theabfm.org.

Robert Cattoi American Board of Family Medicine 